\title{
Convergence of formal CR mappings into strongly pseudoconvex Cauchy-Riemann manifolds
}

\author{
Bernhard Lamel ${ }^{1}$ • Nordine Mir ${ }^{2}$
}

Received: 20 April 2016 / Accepted: 16 June 2017 / Published online: 4 July 2017

(C) The Author(s) 2017. This article is an open access publication

\begin{abstract}
It is shown that any formal holomorphic mapping sending a realanalytic generic submanifold $M \subset \mathbb{C}^{N}$ of finite type into a real-analytic strongly pseudoconvex $\mathrm{CR}$ submanifold $M^{\prime} \subset \mathbb{C}^{N^{\prime}}$ is necessarily convergent. As a consequence, we obtain a positive answer to the long-standing open question of whether formal holomorphic maps sending real-analytic strongly pseudoconvex hypersurfaces into each other are convergent.
\end{abstract}

Mathematics Subject Classification 32V20 - 32V25 - 32V40 - 32H02 . $32 \mathrm{H} 40$

The authors were partially supported by the Qatar National Research Fund, NPRP Project 7-511-1-098. The first author was also supported by the Austrian Science Fund FWF, Project I1776.

$凶$ Bernhard Lamel

bernhard.lamel@univie.ac.at

Nordine Mir

nordine.mir@qatar.tamu.edu

1 Fakultät für Mathematik, Universität Wien, Oskar-Morgenstern-Platz 1, 1090 Wien, Austria

2 Science program, Texas A\&M University at Qatar, PO Box 23874, Education City, Doha, Qatar 


\section{Introduction}

Some of the particular phenomena which occur in CR geometry are the rigidity and the strong regularity properties of CR mappings between CR manifolds. While such properties have very much been studied for automorphisms (see e.g. [2]), many interesting and delicate questions have remained open over the last decades for arbitrary CR mappings between CR manifolds embedded in complex spaces of different dimension. These questions have been at the center of recent interest in the community as shown e.g. by the recent remarkable work on the smooth regularity of CR maps by Berhanu-Xiao [8] (see also the papers $[4,5,11,16,24]$ and the references therein). In this paper, we tackle one such question regarding the convergence of formal holomorphic transformations and prove what seems to be the first general convergence result for formal CR maps in positive codimension.

Recall that, given real-analytic submanifolds $M \subset \mathbb{C}^{N}$ and $M^{\prime} \subset \mathbb{C}^{N^{\prime}}$ through points $p$ and $p^{\prime}$, respectively, a formal holomorphic transformation (or formal holomorphic map), $H:\left(\mathbb{C}^{N}, p\right) \rightarrow\left(\mathbb{C}^{N^{\prime}}, p^{\prime}\right)$ (i.e. an $N^{\prime}$-tuple of formal holomorphic power series at $p$ satisfying $H(p)=p^{\prime}$ ) maps $M$ into $M^{\prime}$ if for any real-analytic function $R$ vanishing on $M^{\prime}$ (near $p^{\prime}$ ), the formal series $R \circ H$ vanishes on $M$; in this case, we write $H(M) \subset M^{\prime}$ or $H:(M, p) \rightarrow\left(M^{\prime}, p^{\prime}\right)$. If, furthermore, $N=N^{\prime}$ and $H$ is invertible, $H$ is called a formal equivalence between $M$ and $M^{\prime}$.

The simplest example of this setting is arguably when one considers formal holomorphic maps of the real line $(\mathbb{R}, 0) \subset(\mathbb{C}, 0)$ into itself. These are given by the formal power series with real coefficients in $\mathbb{R}[[z]]$, and therefore divergent formal maps $H:(\mathbb{R}, 0) \rightarrow(\mathbb{R}, 0)$ not only exist, they actually exist in abundance. This example turns out to be quite misleading for the situation in higher dimensions. Early in the 70s, in their study of the holomorphic equivalence problem for strongly pseudoconvex real-analytic hypersurfaces in $\mathbb{C}^{N}, N \geq 2$, Chern-Moser [9] established that any formal holomorphic invertible transformation between such hypersurfaces must necessarily converge, a conclusion that strongly contrasts with the situation in the complex plane. Since then, there has been a great deal of work in order to identify the optimal geometric conditions on any pair of CR submanifolds $M, M^{\prime} \subset \mathbb{C}^{N}$ ensuring that all their formal equivalences $H:(M, p) \rightarrow\left(M^{\prime}, p^{\prime}\right)$ necessarily converge. In 2002, this convergence problem for formal equivalences was solved in satisfying generality by Baouendi et al. [6] (see also [3] for earlier related results).

On the other hand, the convergence problem for arbitrary formal transformations between real-analytic CR submanifolds $M$ and $M^{\prime}$ embedded in complex spaces of different dimension is much less understood (see e.g. [20,25]). In fact, the following, natural, long-standing question, originating essentially from the 
works of Huang [14,15] and Forstnerič [12] and appearing explicitely e.g. in [25], remained open until now: does Chern-Moser's above mentioned convergence result hold for formal transformations between arbitrary strongly pseudoconvex real-analytic hypersurfaces $M \subset \mathbb{C}^{N}$ and $M^{\prime} \subset \mathbb{C}^{N^{\prime}}$ ? Our current paper gives a positive solution to that question by establishing the following more general result for CR manifolds of any codimension.

Theorem 1.1 Let $M \subset \mathbb{C}^{N}$ and $M^{\prime} \subset \mathbb{C}^{N^{\prime}}$ be real-analytic generic submanifolds, $N, N^{\prime} \geq 2$. Assume that $M$ is of finite type and that $M^{\prime}$ is strongly pseudoconvex. Then, for every $\left(p, p^{\prime}\right) \in M \times M^{\prime}$, any formal holomorphic mapping $H:(M, p) \rightarrow\left(M^{\prime}, p^{\prime}\right)$ is convergent.

Recall here that $M$ is said to be of finite type if the Lie algebra generated by its CR vector fields and its conjugates spans the full complexified tangent space at any point of $M$, and that $M^{\prime}$ is strongly pseudoconvex if it is locally contained in a strongly pseudoconvex hypersurface (see $[2,13])$.

As an immediate consequence of Theorem 1.1, we therefore obtain the above mentioned announced result.

Corollary 1.2 Any formal holomorphic map sending a real-analytic strongly pseudoconvex hypersurface $M \subset \mathbb{C}^{N}$ into another such hypersurface $M^{\prime} \subset$ $\mathbb{C}^{N^{\prime}}, N^{\prime}, N \geq 2$, is convergent.

Besides settling by the affirmative, a long standing open question, Theorem 1.1 appears to be the first general convergence result for formal transformations between real-analytic CR submanifolds in complex spaces of different dimension. Indeed, previous related results either hinged on rather stringent conditions on the maps (see [18]), or assumed some quite restrictive condition on the "codimension" of the mappings (see [22]), or assumed the target manifold to be real-algebraic instead of real-analytic as in [19].

One of the main novelties in our proof of Theorem 1.1 consists of introducing, for any given formal CR map $H: M \rightarrow M^{\prime}$, the notion of "meromorphic infinitesimal deformations" of $H$. These formal objects can be seen as formal meromorphic vector fields tangent to the image of $H$ and are directly related to how degenerate the map $H$ is (see Proposition 4.4). In order to define such infinitesimal deformations, we need to refine several notions of degeneracy used for mappings before in the literature. We should mention that similar objects, suited for the study of the smooth regularity of CR maps, have recently been introduced in the very recent work by Berhanu-Xiao [8]; in the analytic case, for suitably nondegenerate maps, such vector fields also appear in the work of the first author in [18]. However, in our setting, as we are considering arbitrary maps that can be very degenerate, we furthermore need to cope with singularities that generate meromorphic vector fields only generically linearly independent. These infinitesimal deformations can then 
be used to produce a certain type of system of analytic equations satisfied by the mapping $H$, which contains some formal parameters. This is where our second main new ingredient of the present paper comes into play, Proposition 3.1 , which is a very powerful technical convergence result for certain formal power series mappings satisfying certain types of systems of equations.

The paper is organized as follows. We introduce the notations to be used throughout the paper in Sect. 2, followed by the convergence result already alluded to in Sect. 3. We then discuss the notion of a holomorphically degenerate formal CR map and its meromorphic infinitesimal deformations in Sect. 4. Section 5 then establishes two convergence results for CR maps of independent interest, that are used in Sect. 6 to derive the proof of Theorem 1.1.

\section{Power series rings and notations}

Throughout the paper, for $t=\left(t_{1}, \ldots, t_{k}\right) \in \mathbb{C}^{k} \simeq \mathbb{R}^{2 k}$, we denote by $\mathbb{C}[[t, \bar{t}]]$ (resp. $\mathbb{C}\{t, \bar{t}\}$ ) the ring of formal power series (resp. convergent power series) in $t$ and $\bar{t}$ with complex coefficients, where $k \geq 1$. The subring of holomorphic formal power series (resp. holomorphic convergent power series) is denoted by $\mathbb{C}[[t]]$ (resp. $\mathbb{C}\{t\})$. The field of fractions of $\mathbb{C}[[t, \bar{t}]]$ (resp. $\mathbb{C}[[t]]$ ) is denoted by $\mathbb{C}((t, \bar{t}))$ (resp. $\mathbb{C}((t)))$. Note that by complexification $\mathbb{C}[[t, \bar{t}]]$ (resp. $\mathbb{C}\{t, \bar{t}\})$ may be identified with the ring of formal holomorphic power series $\mathbb{C}[[t, \tau]]$ (resp. holomorphic convergent power series $\mathbb{C}\{t, \tau\})$ in $2 k$ complex variables $(t, \tau)$. For a (holomorphic) power series $f(t)=\sum_{\alpha} f_{\alpha} t^{\alpha} \in \mathbb{C}[[t]]$, we shall denote by $\bar{f}(t)$ the formal holomorphic power series in $\mathbb{C}[[t]]$ given by $\bar{f}(t)=$ $\sum_{\alpha} \bar{f}_{\alpha} t^{\alpha}$.

We now briefly recall some notions and facts concerning ideals and rings of formal power series associated to generic submanifolds in complex space. For more details, we refer the reader to e.g. [6,10].

Let $M \subset \mathbb{C}_{Z}^{N}$ be a real-analytic generic submanifold through the origin of codimension $d$. Consider a real-analytic vector-valued defining function $\rho:\left(\mathbb{C}^{N}, 0\right) \rightarrow \mathbb{R}^{d}, \rho=\left(\rho_{1}, \ldots, \rho_{d}\right) \in(\mathbb{C}\{Z, \bar{Z}\})^{d}$, for $M$ near 0 (meaning that $\rho$ is real-valued, $M=\{\rho=0\}$ as germs at 0 and $\left.\partial \rho_{1} \wedge \ldots \wedge \partial \rho_{d}(0) \neq 0\right)$. Denote by $\mathscr{I}(M)$ the ideal in $\mathbb{C}[[Z, \bar{Z}]]$ of formal power series that vanish on $M$ : it is the ideal of formal power series $S(Z, \bar{Z})$ such that for any realanalytic (or formal) parametrization $\psi:\left(\mathbb{R}_{x}^{2 N-d}, 0\right) \rightarrow(M, 0)$, it holds that $S(\psi(x), \bar{\psi}(x))=0$. Note that $\mathscr{I}(M)$ coincides with the ideal generated by $\rho_{1}, \ldots, \rho_{d}$, which we also write as $\left(\rho_{1}, \ldots, \rho_{d}\right)$. It is easy to check that $\mathscr{I}(M)$ is a prime ideal. We define the ring of formal power series on $M$ to be the quotient ring

$$
\mathbb{C}[[M]]:=\mathbb{C}[[Z, \bar{Z}]] / \mathscr{I}(M),
$$


and note that $\mathbb{C}[[M]]$ is an integral domain. For any formal power series $A(Z, \bar{Z})$, we will denote by $\left.A(Z, \bar{Z})\right|_{M}$ the image of $A$ in $\mathbb{C}[[M]]$ under the canonical projection. The field of fractions of $\mathbb{C}[[M]]$ will be denoted by $\mathbb{C}((M))$.

It is also convenient to identify $\mathbb{C}[[M]]$ with the ring of holomorphic power series on the complexification of $M$. Recall that the complexification of $M$, denoted by $\mathscr{M}$, is (the germ at 0 of) the complex submanifold of $\mathbb{C}_{Z}^{N} \times \mathbb{C}_{\zeta}^{N}$ given by the zero-set of the components of the complexified power series mapping $\rho(Z, \zeta)$. If $\mathscr{I}(\mathscr{M})$ denotes the ideal in $\mathbb{C}[[Z, \zeta]]$ of formal holomorphic power series vanishing on $\mathscr{M}$, then we set

$$
\mathbb{C}[[\mathscr{M}]]:=\mathbb{C}[[Z, \zeta]] / \mathscr{I}(\mathscr{M}),
$$

and observe that $\mathbb{C}[[\mathscr{M}]]$ is an integral domain since $\mathscr{I}(\mathscr{M})$ is prime. By analogy with the above, for any formal power series $A(Z, \zeta)$, we will denote by $A(Z, \zeta) \mid \mathscr{M}$ the image of $A$ in $\mathbb{C}[[\mathscr{M}]]$ under the canonical projection.

We may naturally identify $\mathbb{C}[[M]]$ with $\mathbb{C}[[\mathscr{M}]]$ and, note, in particular, the following elementary fact to be used several times in the paper: for any formal power series $A \in \mathbb{C}[[t, \tau]], G_{1}, G_{2} \in(\mathbb{C}[[Z]])^{k}, G_{1}(0)=G_{2}(0)=0$, with $(t, \tau)$ as above, we have

$$
\left.A\left(G_{1}(Z), \overline{G_{2}(Z)}\right)\right|_{M}=\left.0 \Leftrightarrow A\left(G_{1}(Z), \bar{G}_{2}(\zeta)\right)\right|_{\mathscr{M}}=0 .
$$

Let $M$ and $M^{\prime}$ be real-analytic generic submanifolds of $\mathbb{C}^{N}$ and $\mathbb{C}^{N^{\prime}}$, respectively, both passing through the origin, and let $H:\left(\mathbb{C}_{Z}^{N}, 0\right) \rightarrow\left(\mathbb{C}_{Z^{\prime}}^{N^{\prime}}, 0\right)$ be a formal holomorphic mapping. Recall that we say that $H$ sends $M$ into $M^{\prime}$, and write $H(M) \subset M^{\prime}$, or $H: M \rightarrow M^{\prime}$, if for every $A\left(Z^{\prime}, \bar{Z}^{\prime}\right) \in \mathscr{I}\left(M^{\prime}\right)$, the power series $A(H(Z), \overline{H(Z)}) \in \mathscr{I}(M)$. In case $H$ is convergent, the reader can easily check that this means that the germ of $H$ at 0 sends the germ of $M$ (at 0 ) into $M^{\prime}$ in the usual sense.

\section{A convergence result for formal solutions of certain systems of analytic equations}

Our main result in this section provides a very useful and general tool to deduce the convergence of a formal holomorphic map satisfying a type of system of analytic equations when restricted to a generic submanifold of finite type in $\mathbb{C}^{N}$. Recall that a generic submanifold $M \subset \mathbb{C}^{N}$ is of finite type at a point $p \in M$ if the Lie algebra generated by the CR vector fields and their complex conjugates spans the complexified tangent space of $M$ at $p$ (see [2,7]).

Proposition 3.1 Let $M \subset \mathbb{C}^{N}$ be a real-analytic generic submanifold through the origin and $\Theta=\left(\Theta_{1}, \ldots, \Theta_{N^{\prime}}\right)$ be a convergent power series mapping with 
components in $\mathbb{C}\left\{Z, \bar{Z}, \lambda, Z^{\prime}\right\}$ where $Z \in \mathbb{C}^{N}, Z^{\prime} \in \mathbb{C}^{N^{\prime}}, \lambda \in \mathbb{C}^{r}, N^{\prime}, N, r \geq$ 1. Let $H:\left(\mathbb{C}^{N}, 0\right) \rightarrow \mathbb{C}^{N^{\prime}}, G:\left(\mathbb{C}^{N}, 0\right) \rightarrow \mathbb{C}^{r}$ be formal holomorphic power series mappings, vanishing at 0 , satisfying

$$
\left.\Theta(Z, \bar{Z}, \overline{G(Z)}, H(Z))\right|_{M}=0, \quad \text { and }\left.\operatorname{det} \frac{\partial \Theta}{\partial Z^{\prime}}(Z, \bar{Z}, \overline{G(Z)}, H(Z))\right|_{M} \not \equiv 0 .
$$

If $M$ is of finite type at 0 , then $H$ is convergent.

Proposition 3.1 possesses two specific features setting it apart from known results in the literature and making it impossible to deduce its conclusion from these existing classical convergence results for systems of (singular) analytic equations such as e.g. [21, Proposition 4.2]. Firstly, the system of equations is valid when restricted to a certain finite type generic submanifold instead of being valid in the ambient euclidean space. Furthermore, we allow in (3.1) the appearance of a formal power series mapping $G$ that is not related to the solution mapping $H$ and that can be even divergent. The conclusion is nevertheless that the formal mapping $H$ has to converge.

As already pointed out, the result given by Proposition 3.1 is new by itself. Several of the known results from the literature alluded to above can be seen as special cases of Proposition 3.1: when $M$ is a real hypersurface and $N^{\prime}=1$, the result reduces to [22, Proposition 2.2]; and when $M$ is of higher codimension, $N^{\prime}=1$ and $\Theta$ is polynomial in $Z^{\prime}$, it yields the conclusion drawn in [23, Theorem 5.1].

The proof of Proposition 3.1 will be split into two parts. Firstly, in Sect. 3.1 we prove a preliminary result about partial convergence properties of formal power series mappings solutions of certain analytic equations containing formal parameters. Next, in Sect. 3.3, we combine the obtained result with the iterated Segre mapping technique introduced by Baouendi-EbenfeltRothschild [2], which we recall in Sect. 3.2, to prove Proposition 3.1.

\subsection{Partial convergence of formal power series solutions of some systems of formal equations}

Let $f(u, x), g(u, x)$ be two formal power series in $\mathbb{C}[[u, x]], u \in \mathbb{C}^{r_{1}}, x \in \mathbb{C}^{r_{2}}$, $r_{1}, r_{2} \geq 1$. Let $E$ be the subspace of $\mathbb{C}^{r_{1}+r_{2}}$ given by $\{x=0\}$. Given a nonnegative integer $m$, we say that $f(u, x)$ and $g(u, x)$ agree up to order $m$ along $E$ if $\left.\left(\partial^{\beta} f(u, x)-\partial^{\beta} g(u, x)\right)\right|_{E}=0$ in $\mathbb{C}[[u]]$ for every multiindex $\beta \in \mathbb{N}^{r_{1}+r_{2}}$ with $|\beta| \leq m$. We also say that $f$ is convergent along $E$ if $f(u, 0) \in \mathbb{C}\{u\}$.

We start with stating the following lemma going back to [17, Proposition 3.1]. 
Lemma 3.2 Let $P=\left(P_{1}, \ldots, P_{\ell}\right)$ be a formal power series mapping with components in $\mathbb{C}[[u, x, T]]$, where $u \in \mathbb{C}^{r_{1}}, x \in \mathbb{C}^{r_{2}}, T \in \mathbb{C}^{\ell}, \ell, r_{1}, r_{2} \geq 1$. Let $f^{0}(u, x)$ be a $\mathbb{C}^{\ell}$-vector-valued formal power series in $\mathbb{C}[[u, x]]$, vanishing at the origin, satisfying

$$
\mu(u, x):=\operatorname{det} \frac{\partial P}{\partial T}\left(u, x, f^{0}(u, x)\right) \not \equiv 0 .
$$

Fix $\alpha^{0} \in \mathbb{N}^{r_{1}}$ and $\beta^{0} \in \mathbb{N}^{r_{2}}$ such that $\left(\partial_{u}^{\alpha^{0}} \partial_{x}^{\beta^{0}} \mu\right)(0,0) \neq 0$. Then if $m$ is any nonnegative integer and $f(u, x)$ is any $\mathbb{C}^{\ell}$-valued formal power series mapping satisfying

(i) $f(u, x)$ agrees with $f^{0}(u, x)$ at 0 up to order $\left|\alpha^{0}\right|+\left|\beta^{0}\right|$;

(ii) $P\left(u, x, f^{0}(u, x)\right)$ and $P(u, x, f(u, x))$ agree up to order $\left|\beta^{0}\right|+m$ along the subspace $\{x=0\}$,

then $f^{0}(u, x)$ and $f(u, x)$ agree up to order $m$ along the same subspace.

Lemma 3.2 is stated and proved in [17] for a formal power series mapping $P=P(u, T)$ independent of $x$ but the proof given there applies to general power series mappings $P=P(u, x, T)$.

Our next result is the key lemma that will allow us to prove Proposition 3.1. Its proof contains some arguments going back to $[19,21]$.

Lemma 3.3 Let $R=\left(R_{1}, \ldots, R_{\ell}\right)$ be a convergent power series with components in $\mathbb{C}\{s, t, x, \lambda, Y\}$ with $s \in \mathbb{C}^{k_{1}}, t \in \mathbb{C}^{k_{2}}, x \in \mathbb{C}^{k_{3}}, \lambda \in \mathbb{C}^{k_{4}}, Y \in \mathbb{C}^{\ell}$, $\ell, k_{i} \geq 1$. Let $\Delta(s, x)$ and $\psi(s, t, x)$ be respectively $\mathbb{C}^{k_{4}}$-valued and $\mathbb{C}^{\ell}$-valued formal power series mappings, vanishing at the origin, satisfying

$$
\begin{aligned}
& R(s, t, x, \Delta(s, x), \psi(s, t, x))=0, \\
& \eta(s, t, x):=\operatorname{det} \frac{\partial R}{\partial Y}(s, t, x, \Delta(s, x), \psi(s, t, x)) \not \equiv 0 .
\end{aligned}
$$

Assume that all partial derivatives of $\psi$ are convergent along the subspace $F:=\{t=0, x=0\}$. Then for every $\gamma \in \mathbb{N}^{k_{3}}, \partial_{x}^{\gamma} \psi$ is convergent along the subspace $E:=\{x=0\}$.

Proof of Lemma 3.3 Choose $\alpha^{0} \in \mathbb{N}^{k_{1}+k_{2}}$ and $\beta^{0} \in \mathbb{N}^{k_{3}}$ such that $\left(\partial_{(s, t)}^{\alpha^{0}} \partial_{x}^{\beta^{0}} \eta\right)$ $(0,0,0) \neq 0$. We fix an integer $m \geq\left|\alpha^{0}\right|$ and shall prove that for every $\gamma \in \mathbb{N}^{k_{3}}$ with $|\gamma| \leq m, \partial_{x}^{\gamma} \psi$ is convergent along $E$.

Write $\Delta(s, x)=\sum_{\delta \in \mathbb{N}^{k_{3}}} \Delta_{\delta}(s) x^{\delta}$ and $\psi(s, t, x)=\sum_{\delta \in \mathbb{N}^{k_{3}}} \psi_{\delta}(s, t) x^{\delta}$. From (3.2) and the chain rule, for every $\beta \in \mathbb{N}^{k_{3}}$ with $|\beta| \leq m+\left|\beta^{0}\right|$, there exists a $\mathbb{C}^{\ell}$-valued power series mapping $R^{\beta}$, depending only on $R$ (and not on $\Delta$ nor $\psi$ ) such that 


$$
\begin{aligned}
0 & =\left.\frac{\partial^{|\beta|}}{\partial x^{\beta}}(R(s, t, x, \Delta(s, x), \psi(s, t, x)))\right|_{x=0} \\
& =R^{\beta}\left(s, t,\left(\Delta_{\delta}(s)\right)_{|\delta| \leq m+\left|\beta^{0}\right|},\left(\psi_{\delta}(s, t)\right)_{|\delta| \leq m+\left|\beta^{0}\right|}\right) .
\end{aligned}
$$

We note that it follows from the chain rule that every component of the mapping $R^{\beta}$ belongs to the ring $\mathbb{C}\{s, t, \lambda, Y\}\left[\left(\lambda_{\delta}\right)_{1 \leq|\delta| \leq m+\left|\beta^{0}\right|},\left(Y_{\delta}\right)_{1 \leq|\delta| \leq m+\left|\beta^{0}\right|}\right]$, where $\lambda_{\delta} \in \mathbb{C}^{k_{4}}$ and $Y_{\delta} \in \mathbb{C}^{\ell}$ for every $\delta \in \mathbb{N}^{k_{3}}$.

In what follows we write $\lambda_{0}:=\lambda$ and $Y_{0}:=Y$. Thanks to (3.3), we may view the formal mapping $\lambda_{\delta}=\Delta_{\delta}(s),|\delta| \leq m+\left|\beta^{0}\right|$, as a formal solution of the system of formal equations

$$
R^{\beta}\left(s, t,\left(\lambda_{\delta}\right)_{|\delta| \leq m+\left|\beta^{0}\right|},\left(\psi_{\delta}(s, t)\right)_{|\delta| \leq m+\left|\beta^{0}\right|}\right)=0, \quad|\beta| \leq m+\left|\beta^{0}\right| .
$$

As the solution mapping of (3.4) is independent of $t, \lambda_{\delta}=\Delta_{\delta}(s),|\delta| \leq$ $m+\left|\beta^{0}\right|$ also satisfies the equivalent system of equations

$$
\begin{aligned}
& \left.\left(\frac{\partial^{|v|}}{\partial t^{v}}\left\{R^{\beta}\left(s, t,\left(\lambda_{\delta}\right)_{|\delta| \leq m+\left|\beta^{0}\right|},\left(\psi_{\delta}(s, t)\right)_{|\delta| \leq m+\left|\beta^{0}\right|}\right)\right\}\right)\right|_{t=0}=0 \\
& |\beta| \leq m+\left|\beta^{0}\right|, \quad v \in \mathbb{N}^{k_{2}}
\end{aligned}
$$

As all partial derivatives of $\psi$ are convergent along $F$, (3.5) becomes a system of complex-analytic equations and, therefore, by Artin's approximation theorem [1], there exists a convergent mapping

$$
\left(\lambda_{\delta}\right)_{|\delta| \leq m+\left|\beta^{0}\right|}=\left(\widetilde{\Delta}_{\delta}(s)\right)_{|\delta| \leq m+\left|\beta^{0}\right|}
$$

which agrees with the original formal solution $\left(\Delta_{\delta}(s)\right)_{|\delta| \leq m+\left|\beta^{0}\right|}$ up to order $\left|\alpha^{0}\right|$ and which satisfies (3.5), and therefore, also (3.4). From the definition of the power series mapping $R^{\beta}$, we see that this implies

$$
\begin{aligned}
0 & =R^{\beta}\left(s, t,\left(\widetilde{\Delta}_{\delta}(s)\right)_{|\delta| \leq m+\left|\beta^{0}\right|},\left(\psi_{\delta}(s, t)\right)_{|\delta| \leq m+\left|\beta^{0}\right|}\right) \\
& =\left.\frac{\partial^{|\beta|}}{\partial x^{\beta}}(R(s, t, x, \widetilde{\Delta}(s, x), \psi(s, t, x)))\right|_{x=0},|\beta| \leq m+\left|\beta^{0}\right|,
\end{aligned}
$$

where $\widetilde{\Delta}(s, x)=\sum_{|\delta| \leq m+\left|\beta^{0}\right|} \widetilde{\Delta}_{\delta}(s) x^{\delta}$. Since for every $|\delta| \leq m+\left|\beta^{0}\right|$, $\Delta_{\delta}(s)$ and $\widetilde{\Delta}_{\delta}(s)$ agree up to order $\left|\alpha^{0}\right|$ for every $\delta$ with $|\delta| \leq m+\left|\beta^{0}\right|$, we have $\left(\partial_{s}^{\alpha} \partial_{x}^{\beta} \Delta\right)(0,0)=\left(\partial_{s}^{\alpha} \partial_{x}^{\beta} \widetilde{\Delta}\right)(0,0)$ for $|\alpha| \leq\left|\alpha^{0}\right|$ and $|\beta| \leq\left|\beta^{0}\right|$. Since $\left(\partial_{(s, t)}^{\alpha^{0}} \partial_{x}^{\beta^{0}} \eta\right)(0,0,0) \neq 0$, we get 


$$
\begin{aligned}
\left(\partial_{(s, t)}^{\alpha^{0}} \partial_{x}^{\beta^{0}} \tilde{\eta}\right)(0,0,0) & \neq 0, \\
\text { where } \tilde{\eta}(s, t, x): & =\operatorname{det} \frac{\partial R}{\partial Y}(s, t, x, \widetilde{\Delta}(s, x), \psi(s, t, x)) .
\end{aligned}
$$

On the other hand, from (3.6), we see that $Y_{\delta}=\psi_{\delta}(s, t)$ for $|\delta| \leq m+\left|\beta^{0}\right|$ is a formal solution of the system of complex-analytic equations

$$
R^{\beta}\left(s, t,\left(\widetilde{\Delta}_{\delta}(s)\right)_{|\delta| \leq m+\left|\beta^{0}\right|},\left(Y_{\delta}\right)_{|\delta| \leq m+\left|\beta^{0}\right|}\right)=0,|\beta| \leq m+\left|\beta^{0}\right| .
$$

By applying Artin's approximation theorem [1], there exists a convergent solution mapping $Y_{\delta}=\widetilde{\psi}_{\delta}(s, t),|\delta| \leq m+\left|\beta^{0}\right|$, of (3.8) that agrees with the above mentioned formal solution up to order $\left|\alpha^{0}\right|+\left|\beta^{0}\right|$. Set $\widetilde{\psi}(s, t, x)=\sum_{|\delta| \leq m+\left|\beta^{0}\right|} \widetilde{\psi}_{\delta}(s, t) x^{\delta}$. Then, from our construction, $R(s, t, \widetilde{\Delta}(s, x), \psi(s, t, x))$ and $R(s, t, \widetilde{\Delta}(s, x), \widetilde{\psi}(s, t, x))$ agree up to order $m+\left|\beta^{0}\right|$ along the subspace $\{x=0\}$. Furthermore since $m \geq\left|\alpha^{0}\right|, \psi(s, t, x)$ and $\widetilde{\psi}(s, t, x)$ agree at 0 up to order $\left|\alpha^{0}\right|+\left|\beta^{0}\right|$. Hence, in view of (3.7), we may apply Lemma 3.2 to conclude that $\psi(s, t, x)$ and $\widetilde{\psi}(s, t, x)$ agree up to order $m$ along the subspace $\{x=0\}$. Hence for every $\gamma \in \mathbb{N}^{k_{3}}$ with $|\gamma| \leq m$, $\left.\left(\partial_{x}^{\gamma} \psi\right)\right|_{x=0}=\gamma ! \widetilde{\psi}_{\gamma}$ is convergent. The proof of Lemma 3.3 is complete.

Remark 3.4 From the above proof, it is clear that the conclusion of Lemma 3.3 still holds if all partial derivatives of $\psi$ are assumed to be convergent along a more general formal submanifold of the form $\{t=A(s), x=0\}$ instead of the subspace $F$, where $A$ is any formal power series in $\mathbb{C}[[s]]$ vanishing at the origin.

\subsection{Segre set mappings and basic properties}

Let $M \subset \mathbb{C}^{N}$ be a connected generic real-analytic submanifold through the origin of CR dimension $n$ and codimension $d, N=n+d$. There exists normal local holomorphic coordinates $Z=(z, w) \in \mathbb{C}^{n} \times \mathbb{C}^{d}$ such that $M$ is given near the origin by an equation of the form

$$
w=Q(z, \bar{z}, \bar{w}),
$$

where $Q$ is a $\mathbb{C}^{d}$-valued holomorphic map near $0 \in \mathbb{C}^{n+N}$ (see e.g. [2]). As a real-analytic submanifold, we may complexify $M$ to get to its so-called complexification $\mathscr{M}$ as already discussed in Sect. 2. The germ of the complex submanifold $\mathscr{M}$ of $\mathbb{C}^{2 N}$ at 0 is given by

$$
\left\{(Z, \zeta) \in\left(\mathbb{C}^{N} \times \mathbb{C}^{N}, 0\right): w=Q(z, \chi, \tau)\right\},
$$


where we write $Z=(z, w) \in \mathbb{C}^{n} \times \mathbb{C}^{d}$ and $\zeta=(\chi, \tau) \in \mathbb{C}^{n} \times \mathbb{C}^{d}$. That we have chosen normal coordinates means that we have the following two identities:

$$
Q(z, 0, \tau)=Q(0, \chi, \tau)=\tau, \quad Q(z, \chi, \bar{Q}(\chi, z, w))=w .
$$

We now define the iterated Segre mappings attached to $M$ near the origin (see e.g. [2]). For any nonnegative integer $j$, we denote by $t^{j}$ a variable lying in $\mathbb{C}^{n}$ and also introduce the variable $t^{[j]}:=\left(t^{1}, \ldots, t^{j}\right) \in \mathbb{C}^{n j}$. For $x \in \mathbb{C}^{d}$ sufficiently close to 0 we set

$$
V_{0}(x):=(0, x) \in \mathbb{C}^{N}
$$

and also define the map $V_{j}:\left(\mathbb{C}^{n j} \times \mathbb{C}^{d}, 0\right) \rightarrow \mathbb{C}^{N}$ for $j \geq 1$ inductively as follows:

$V_{j}\left(t^{[j]}, x\right):=\left(t^{j}, U_{j}\left(t^{[j]}, x\right)\right)$, where $U_{j}\left(t^{[j]}, x\right):=Q\left(t^{j}, \bar{V}_{j-1}\left(t^{[j-1]}, x\right)\right)$.

Each iterated Segre mapping $V_{j}$ clearly defines a holomorphic map in a neighbhorhood of 0 in $\mathbb{C}^{n j+d}$. Note that if $x \in \mathbb{R}^{d}$, the map $V_{j}(\cdot, x)$ parametrizes the "usual" Segre set of order $j$ attached to the point $(0, x) \in M$ (see e.g. [2]). From (3.11), we have the following useful identities

$$
V_{j}(0, x)=(0, x),\left.\quad V_{j+2}\left(t^{[j+2]}, x\right)\right|_{t^{j+2}=t^{j}}=V_{j}\left(t^{[j]}, x\right), \quad j \geq 0,
$$

and for every $j \geq 0$, the germ at 0 of the holomorphic map $\left(V_{j+1}, \bar{V}_{j}\right)$ takes its values in the complexification $\mathscr{M}$.

We will need the following fact about the iterated Segre mappings whose proof uses the chain rule and the fact that the matrix $\frac{\partial U_{j}}{\partial x}(0)$ has rank $d$, where $U_{j}$ is given by (3.13) (for the proof see e.g. [17, Corollary 6.7]).

Lemma 3.5 Let $h \in \mathbb{C}[[Z]]$ and $j \geq 1$. Iffor some integer $m$, all power series $\partial_{x}^{\gamma}\left(h \circ V_{j}\right)$ are convergent along $\{x=0\}$ for $\gamma \in \mathbb{N}^{d}$ with $|\gamma| \leq m$, then for all $\alpha \in \mathbb{N}^{N}$ and $\beta \in \mathbb{N}^{d}$ with $|\alpha|+|\beta| \leq m, \partial_{x}^{\beta}\left(\left(\partial_{Z}^{\alpha} h\right) \circ V_{j}\right)$ is convergent along $\{x=0\}$.

We will also need the following characterization of the finite type condition in terms of the iterated Segre mappings due to Baouendi-Ebenfelt-Rothschild (see e.g. [2]):

Theorem 3.6 With the above notation, the generic submanifold $M \subset \mathbb{C}^{N}$ is of finite type at 0 if and only if there exists a positive integer $\ell_{0}, 2 \leq \ell_{0} \leq 2(d+1)$, such that in any neighborhood $U$ of 0 in $\mathbb{C}^{n \ell_{0}}$ there exists $t_{0}^{\left[\ell_{0}\right]} \in U$ such that 


$$
\operatorname{rk} \frac{\partial V_{\ell_{0}}}{\partial t^{\left[\ell_{0}\right]}}\left(t_{0}^{\left[\ell_{0}\right]}, 0\right)=N, \quad V_{\ell_{0}}\left(t_{0}^{\left[\ell_{0}\right]}, 0\right)=0
$$

\subsection{Proof of Proposition 3.1}

We choose normal coordinates $Z=(z, w) \in \mathbb{C}^{n} \times \mathbb{C}^{d}$ for $M$ near 0 as given in Sect. 3.2. Complexifying (3.1), we get

$$
\left.\Theta(Z, \zeta, \bar{G}(\zeta), H(Z))\right|_{\mathscr{M}}=0, \quad \text { and }\left.\operatorname{det} \frac{\partial \Theta}{\partial Z^{\prime}}(Z, \zeta, \bar{G}(\zeta), H(Z))\right|_{\mathscr{M}} \not \equiv 0
$$

For every integer $j \geq 1$, the germ at $0 \in \mathbb{C}^{n(j+1)} \times \mathbb{C}^{d}$ of the holomorphic map $\left(V_{j+1}, \bar{V}_{j}\right)$ is submersive onto $\mathscr{M}$ at 0 . Hence, for $j \geq 1$, (3.16) implies

$$
\begin{aligned}
& \Theta\left(V_{j+1}, \bar{V}_{j}, \bar{G} \circ \bar{V}_{j}, H \circ V_{j+1}\right)=0, \quad \text { and } \\
& \operatorname{det} \frac{\partial \Theta}{\partial Z^{\prime}}\left(V_{j+1}, \bar{V}_{j}, \bar{G} \circ \bar{V}_{j}, H \circ V_{j+1}\right) \not \equiv 0,
\end{aligned}
$$

where the previous identities hold in the ring $\mathbb{C}\left[\left[t^{[j+1]}, x\right]\right]$. We now prove the following convergence propagation property :

Claim Let $j \geq 1$. If, for every $\alpha \in \mathbb{N}^{N},\left(\partial_{Z}^{\alpha} H\right) \circ V_{j-1}$ is convergent along the subspace $\{x=0\}$, then for every $\alpha \in \mathbb{N}^{N},\left(\partial_{Z}^{\alpha} H\right) \circ V_{j+1}$ is convergent along $\{x=0\}$.

We now prove the claim. Fix $j \geq 1$. Set $R\left(t^{[j+1]}, x, \lambda, Z^{\prime}\right):=$ $\Theta\left(V_{j+1}, \bar{V}_{j}, \lambda, Z^{\prime}\right)$ whose components are convergent power series, and $\Delta:=\bar{G} \circ \bar{V}_{j}$ and $\psi:=H \circ V_{j+1}$. From the assumption in the claim and the second identity in (3.14), we see that all partial derivatives of $\psi$ are convergent along the subspace $\left\{t^{j+1}=t^{j-1}, x=0\right\}$. Hence (3.17) together with Lemma 3.3 and Remark 3.4 yield that for every $\gamma \in \mathbb{N}^{d}, \partial_{x}^{\gamma} \psi=\partial_{x}^{\gamma}\left(H \circ V_{j+1}\right)$ is convergent along $\{x=0\}$. By Lemma 3.5, this implies that for every $\alpha \in \mathbb{N}^{N}$, $\left(\partial_{Z}^{\alpha} H\right) \circ V_{j+1}$ is convergent along $\{x=0\}$, which proves the claim.

We may now finish the proof of Proposition 3.1. We first notice that, in view of (3.12), for every $\alpha \in \mathbb{N}^{N},\left(\partial_{Z}^{\alpha} H\right) \circ V_{0}$ is convergent along $\{x=0\}$. Hence using the claim we get that for every even integer $j$ and for every $\alpha \in \mathbb{N}^{N}$, $\left(\partial_{Z}^{\alpha} H\right) \circ V_{j}$ is convergent along $\{x=0\}$. By Theorem 3.6, we may choose an integer $\ell_{0}$, that may be assumed to be even, satisfying the conclusion of that theorem. Set $v_{\ell_{0}}:=V_{\ell_{0}}(\cdot, 0)$. As $H \circ v_{\ell_{0}}$ is convergent in some open neighborhood $U$ of 0 in $\mathbb{C}^{n \ell_{0}}$, we may pick $t_{0}^{\left[\ell_{0}\right]} \in U$ and, thanks to (3.15), find a germ of a holomorphic map $A:\left(\mathbb{C}^{N}, 0\right) \rightarrow\left(\mathbb{C}^{n \ell_{0}}, t_{0}^{\left[\ell_{0}\right]}\right)$ such that $v_{\ell_{0}} \circ A=\operatorname{Id}_{\mathbb{C}^{N}}$ near the origin. Hence $H=\left(H \circ v_{\ell_{0}}\right) \circ A$ is convergent. This completes the proof of Proposition 3.1. 


\section{Degeneracy of formal CR maps and their formal meromorphic infinitesimal deformations}

In this section, we introduce the notion of holomorphically degenerate and nondegenerate formal maps between real-analytic generic submanifolds in complex space, generalizing the notion of constantly degenerate maps introduced by the first author in [18]. For holomorphically degenerate maps, we shall prove, in Proposition 4.4, an important property relating the so-called "generic degeneracy" of the map with the dimension of the space of their formal meromorphic infinitesimal deformations. In what follows, we give the precise definitions of the notions we'll need for the rest of this paper.

Let $M \subset \mathbb{C}^{N}$ and $M^{\prime} \subset \mathbb{C}^{N^{\prime}}$ be real-analytic generic submanifolds through the origin of $\mathrm{CR}$ dimension $n, n^{\prime}$ and codimension $d, d^{\prime}$ respectively, $N, N^{\prime} \geq 2, n \geq 1$. Consider $H:\left(\mathbb{C}_{Z}^{N}, 0\right) \rightarrow\left(\mathbb{C}_{Z^{\prime}}^{N^{\prime}}, 0\right)$ a formal holomorphic map sending $M$ into $M^{\prime}$ and let $\rho^{\prime}=\left(\rho_{1}^{\prime}, \ldots, \rho_{d^{\prime}}^{\prime}\right)$ be a real-analytic $\mathbb{R}^{d^{\prime}}$ valued defining function for $M^{\prime}$ near 0 such that $\partial \rho_{1}^{\prime} \wedge \ldots \wedge \partial \rho_{d^{\prime}}^{\prime}(0) \neq 0$. Let $\bar{L}_{1}, \ldots, \bar{L}_{n}$ be a basis of real-analytic CR vector fields for $M$ near 0 . For every integer $k$, we define the following $\mathbb{C}((M))$-vector subspace of $\mathbb{C}((M))^{N^{\prime}}$

$$
\mathscr{E}_{k}(H):=\operatorname{Span}_{\mathbb{C}((M))}\left\{E_{j}^{\alpha}: \alpha \in \mathbb{N}^{n},|\alpha| \leq k, 1 \leq j \leq d^{\prime}\right\},
$$

where

$$
E_{j}^{\alpha}:=\left(E_{j, \ell}^{\alpha}\right)_{1 \leq \ell \leq N^{\prime}}=\left(\left.\bar{L}^{\alpha} \rho_{j, Z_{1}^{\prime}}^{\prime}(H, \bar{H})\right|_{M}, \ldots,\left.\bar{L}^{\alpha} \rho_{j, Z_{N}^{\prime}}^{\prime}(H, \bar{H})\right|_{M}\right) \in \mathbb{C}[[M]]^{N^{\prime}}
$$

Set $\mu_{k}^{H}:=\operatorname{dim}_{\mathbb{C}((M))} \mathscr{E}_{k}(H)$. The reader can check that the subspace $\mathscr{E}_{k}(H)$ is independent of the choice of the basis of real-analytic CR vector fields and of the choice of the vector-valued defining function $\rho^{\prime}$ for $M^{\prime}$. It is also clear that the sequence $\left(\mu_{k}^{H}\right)$ strictly increases then stabilizes at some index $k_{0}$ i.e. $\mu_{k}^{H}=\mu_{k_{0}}^{H}$ for $k \geq k_{0}$ and $\mu_{k_{0}-1}^{H}<\mu_{k_{0}}^{H}$. In what follows we write $\mu^{H}:=\mu_{k_{0}}^{H}$.

Definition 4.1 Let $H, M, M^{\prime}$ be as above.

(a) We define the generic degeneracy of $H$ as $\kappa^{H}:=N^{\prime}-\mu^{H}$.

(b) We say that $H$ is a holomorphically nondegenerate formal holomorphic map if $\kappa^{H}=0$.

Remark 4.2 We remark the following three facts.

(i) From the fact that $M^{\prime}$ is a generic submanifold of $\mathbb{C}^{N^{\prime}}$, one can easily check that the generic degeneracy of a formal holomorphic map always satisfies $\kappa^{H} \leq n^{\prime}$, where $n^{\prime}$ is the CR dimension of $M^{\prime}$.

(ii) Definition 4.1 contains and generalizes the well-known notion of holomorphically nondegenerate generic submanifold. Indeed, if one takes 
$M=M^{\prime}$ and $H$ to be the identity mapping, then $M$ is holomorphically nondegenerate in the sense of Stanton [26] if and only if the identity mapping is holomorphically nondegenerate in the sense of Definition 4.1. The reader is referred to [2, Chapter XI] for the details of the proof of such a statement.

(iii) A formal map $H: M \rightarrow M^{\prime}$ is said to be of constant degeneracy (as in [18]) if

$$
\operatorname{dim} \operatorname{Span}_{\mathbb{C}}\left\{\left.E_{j}^{\alpha}\right|_{0}: \alpha \in \mathbb{N}^{n}, 1 \leq j \leq d^{\prime}\right\}=\mu^{H}
$$

As pointed out above, we will show that $\kappa^{H}$ can be interpreted as the dimension of a space of (meromorphic) vector fields defined along the image of $M$ under $H$; formally, we define:

Definition 4.3 Let $H, M, M^{\prime}$ be as above and $V=\left(V_{1}, \ldots, V_{N^{\prime}}\right) \in$ $\mathbb{C}((Z))^{N^{\prime}}$. We say that $V$ is a formal meromorphic infinitesimal deformation of $H$ if $V$ is tangent to $M^{\prime}$ along $H(M)$ i.e. if

$$
\forall j \in\left\{1, \ldots, d^{\prime}\right\},\left.\quad \sum_{r=1}^{N^{\prime}}\left(V_{r} \rho_{j, Z_{r}^{\prime}}^{\prime}(H, \bar{H})\right)\right|_{M}=0 \text { in } \mathbb{C}((M)) .
$$

The next proposition is our main result of this section. We should point out that, in the case of constant degeneracy maps (i.e with "no singularities"), this dates back to [18], and it has also recently appeared in the context of smooth CR mappings of constant degeneracy in the work of Berhanu-Xiao [8].

Proposition 4.4 Let $M \subset \mathbb{C}^{N}$ and $M^{\prime} \subset \mathbb{C}^{N^{\prime}}$ be real-analytic generic submanifolds through the origin and $H:\left(\mathbb{C}_{Z}^{N}, 0\right) \rightarrow\left(\mathbb{C}_{Z^{\prime}}^{N^{\prime}}, 0\right)$ be a formal holomorphic map sending $M$ into $M^{\prime}$. Assume that $H$ is a holomorphically degenerate map of generic degeneracy $\kappa:=\kappa^{H}>0$. Then, interchanging the $Z^{\prime}$-coordinates if necessary, and using the above notation, we may split $Z^{\prime}=\left(\widetilde{Z}^{\prime}, \widehat{Z}^{\prime}\right) \in \mathbb{C}^{N^{\prime}-\kappa} \times \mathbb{C}^{\kappa}$ so that the following holds:

(i) $\operatorname{Span}_{\mathbb{C}((M))}\left\{\widetilde{E}_{j}^{\alpha}: \alpha \in \mathbb{N}^{n}, 1 \leq j \leq d^{\prime}\right\}=\mathbb{C}((M))^{N^{\prime}-\kappa}$ where we write $E_{j}^{\alpha}=\left(\widetilde{E}_{j}^{\alpha}, \widehat{E}_{j}^{\alpha}\right)$;

(ii) For $v=N^{\prime}-\kappa+1, \ldots, N^{\prime}$, there exists a formal meromorphic infinitesimal deformation of $H, X^{v}=\left(X_{1}^{v}, \ldots, X_{N^{\prime}}^{v}\right) \in \mathbb{C}((Z))^{N^{\prime}}$, satisfying $X_{i}^{v}=\delta_{i \nu}$ for $N^{\prime}-\kappa+1 \leq i \leq N^{\prime}$.

Proposition 4.4 shows that every holomorphically degenerate formal mapping $H$, sending $M$ into $M^{\prime}$, of generic degeneracy $\kappa$, generates $\kappa$ linearly independent (over $\mathbb{C}((Z))$ ) formal meromorphic infinitesimal deformations of 
$H$ (or, after clearing denominators, formal holomorphic infinitesimal deformations). In the case where $M=M^{\prime}$ and $H$ is the identity mapping, Proposition 4.4 essentially boils down to Stanton's criterion for holomorphically degenerate generic submanifolds (see e.g. [2,26]).

Though the proof given in [18] can be adapted to the present setting, as also the proof of the result analogous to Proposition 4.4 given in the smooth case in [8], we shall rather give here a simpler proof of Proposition 4.4 that is based on the following elementary linear algebra lemma.

Lemma 4.5 Let $A(s, t):=\left(A^{1}(s, t), \ldots, A^{r}(s, t)\right)$ be a $m \times r$ matrix with coefficients in $\mathbb{C}((s, t))$ where $s \in \mathbb{C}^{k_{1}}, t \in \mathbb{C}^{k_{2}}, m, r, k_{1}, k_{2} \geq 1$. Write $A^{j}=\left(A_{1}^{j}, \ldots, A_{m}^{j}\right)^{T}$ as a column vector for $1 \leq j \leq r$. Assume that :

(i) The rank over $\mathbb{C}((s, t))$ of the matrix $A$ equals $\ell_{0}<m$, and that its first $\ell_{0}$ rows of are linearly independent over $\mathbb{C}((s, t))$.

(ii) The rank over $\mathbb{C}((s, t))$ of the $m \times r\left(k_{1}+1\right)$ matrix $\left(A, \partial_{s} A\right)$ is also $\ell_{0}$. Then there exists a $\left(m-\ell_{0}\right) \times \ell_{0}$ matrix $B=\left(B_{i \ell}\right)$ with coefficients in $\mathbb{C}((t))$ such that

$$
A_{i}^{j}=\sum_{\ell=1}^{\ell_{0}} B_{i \ell} A_{\ell}^{j}, 1+\ell_{0} \leq i \leq m, 1 \leq j \leq r .
$$

Proof of Lemma 4.5 From (i), there exists a matrix $B=\left(B_{i \ell}\right), \ell_{0}+1 \leq i \leq m$, $1 \leq \ell \leq \ell_{0}$ with coefficients in $\mathbb{C}((s, t))$ such that (4.3) holds. In order to prove the lemma, we need to check that $\partial_{s} B_{i \ell}=0$ for $i, \ell$ as above. A simple row manipulation shows that the rank of the matrix $\left(A, \partial_{s} A\right)$ is the same as of that of the following matrix:

$$
\Upsilon=\left[\begin{array}{ccc|ccc}
A_{1}^{1} & \ldots & A_{1}^{r} & \partial_{s} A_{1}^{1} & \ldots & \partial_{s} A_{1}^{r} \\
\vdots & \ldots & \vdots & \vdots & \ldots & \vdots \\
A_{\ell_{0}}^{1} & \ldots & A_{\ell_{0}}^{r} & \partial_{s} A_{\ell_{0}}^{1} & \ldots & \partial_{s} A_{\ell_{0}}^{r} \\
\hline & & & & \\
& & & &
\end{array}\right]
$$

where $C$ is a $\left(m-\ell_{0}\right) \times r k_{1}$ matrix whose row vectors $C_{\ell_{0}+1}, \ldots C_{m}$ are given by

$$
C_{i}=\left(\partial_{s} A_{i}^{1}-\sum_{1 \leq \ell \leq \ell_{0}} B_{i \ell} \partial_{s} A_{\ell}^{1}, \ldots, \partial_{s} A_{i}^{r}-\sum_{1 \leq \ell \leq \ell_{0}} B_{i \ell} \partial_{s} A_{\ell}^{r}\right), \quad 1+\ell_{0} \leq i \leq m .
$$

By (ii), the rank of the matrix $\Upsilon$ is the same as that of $A$. In view of (i), this implies that $C=0$, i.e. for every $1+\ell_{0} \leq i \leq m, 1 \leq j \leq r$ 


$$
\partial_{s} A_{i}^{j}=\sum_{\ell \leq \ell_{0}} B_{i \ell} \partial_{s} A_{\ell}^{j} .
$$

But (4.3) together with (4.5) imply that for all $i, j$ as above

$$
\sum_{\ell \leq \ell_{0}} \partial_{s} B_{i \ell} A_{\ell}^{j}=0
$$

By (ii), the $\ell_{0}$ first rows of the matrix $A$ are linearly independent over $\mathbb{C}((s, t))$ and hence, from (4.6), we get that $\partial_{s} B_{i \ell}=0$, for $1+\ell_{0} \leq i \leq m, 1 \leq \ell \leq \ell_{0}$, which completes the proof of the lemma.

We now show that Proposition 4.4 is a direct application of Lemma 4.5.

Proof of Proposition 4.4 Let $M, M^{\prime}, H$ be as in the proposition and we follow the notation previously defined. We choose normal coordinates $Z=(z, w) \in$ $\mathbb{C}^{n} \times \mathbb{C}^{d}$ for $M$ as defined in Sect. 3.2. We write $Q=\left(Q^{(1)}, \ldots, Q^{(d)}\right)$ and $w=\left(w_{1}, \ldots, w_{d}\right)$. Consider the following basis of CR vector fields of $M$ near 0

$$
\bar{L}_{j}:=\frac{\partial}{\partial \bar{z}_{j}}-\sum_{k=1}^{d} \bar{Q}_{\bar{z}_{j}}^{(k)}(\bar{z}, z, w) \frac{\partial}{\partial \bar{w}_{k}}, j=1, \ldots, n .
$$

Let $\mathscr{M}$ be the complexification of $M$ as given in (3.10). The $\mathrm{CR}$ vector fields given by (4.7) may be complexified to give rise to the following holomorphic vector fields tangent to $\mathscr{M}$

$$
\mathscr{L}_{j}:=\frac{\partial}{\partial \chi_{j}}-\sum_{k=1}^{d} \bar{Q}_{\chi_{j}}^{(k)}(\chi, z, w) \frac{\partial}{\partial \tau_{k}}, j=1, \ldots, n .
$$

Here $\zeta=(\chi, \tau) \in \mathbb{C}^{n} \times \mathbb{C}^{d}, \tau=\left(\tau_{1}, \ldots, \tau_{d}\right)$ and $(Z, \chi)$ can be seen as local holomorphic coordinates for $\mathscr{M}$. For $\alpha \in \mathbb{N}^{n},|\alpha| \leq k$ and $1 \leq j \leq d^{\prime}$, the power series maps $E_{j}^{\alpha}$ defined in (4.2), which generate the subspace $\mathscr{E}_{k}(H) \subset$ $\mathbb{C}((M))^{N^{\prime}}$ introduced in (4.1) over $\mathbb{C}((M))$, can be complexified to give power series mappings in $\mathbb{C}[[\mathscr{M}]]$ that we will denote by $\mathbb{E}_{j}^{\alpha}$. With our choice of vector fields given by (4.7) and (4.8), we have for $\alpha \in \mathbb{N}^{n}, 1 \leq j \leq d^{\prime}$ and $1 \leq \ell \leq N^{\prime}$

$$
\begin{aligned}
\mathbb{E}_{j, \ell}^{\alpha}=\mathbb{E}_{j, \ell}^{\alpha}(Z, \chi) & =\left.\mathscr{L}^{\alpha} \rho_{j, Z_{\ell}^{\prime}}^{\prime}(H(Z), \bar{H}(\zeta))\right|_{\tau=\bar{Q}(\chi, Z)} \\
& =\frac{\partial^{\alpha}}{\partial \chi^{\alpha}}\left(\rho_{j, Z_{\ell}^{\prime}}^{\prime}(H(Z), \bar{H}(\chi, \bar{Q}(\chi, Z)))\right. \\
& =\partial_{\chi}^{\alpha} \mathbb{E}_{j, \ell}^{0} .
\end{aligned}
$$


As $H$ is of generic degeneracy $\kappa$, it is obvious that, interchanging the components of $Z^{\prime}$ if necessary, we may split $Z^{\prime}=\left(\widetilde{Z}^{\prime}, \widehat{Z}^{\prime}\right) \in \mathbb{C}^{N^{\prime}-\kappa} \times \mathbb{C}^{\kappa}$ so that (i) holds. In view of (4.9), this means that there exists an integer $k_{0}$ such that the matrix $A$ formed by the column vectors $\partial_{\chi}^{\alpha} \mathbb{E}_{j}^{0}$ in $\mathbb{C}[[Z, \chi]]^{N^{\prime}}$ for $\alpha \in \mathbb{N}^{n}$, $|\alpha| \leq k_{0}$ and $1 \leq j \leq d^{\prime}$ is of rank $N^{\prime}-\kappa<N^{\prime}$ (over $\left.\mathbb{C}((Z, \chi))\right)$ and that the rank of the matrix $\left(A, \partial_{\chi} A\right)$ is also of rank $N^{\prime}-\kappa$. We may apply Lemma 4.5 to conclude that any $v$-th row vector of the matrix $A$, with $N^{\prime}-\kappa+1 \leq v \leq N^{\prime}$ can be written as a linear combination of the $N^{\prime}-\kappa$ first rows of $A$ with coefficients in $\mathbb{C}((Z))$. This implies, in particular, that for $1 \leq j \leq d^{\prime}$ and $N^{\prime}-\kappa+1 \leq v \leq N^{\prime}$, we have the following identity in $\mathbb{C}((Z, \chi))$

$$
\mathbb{E}_{j, v}^{0}=\sum_{\ell=1}^{N^{\prime}-\kappa} X_{\ell}^{v} \mathbb{E}_{j, \ell}^{0}
$$

where each $X_{\ell}^{\nu} \in \mathbb{C}((Z))$ for $1 \leq \ell \leq N^{\prime}-\kappa$ and $N^{\prime}-\kappa+1 \leq \nu \leq N^{\prime}$. Equivalently, we have in $\mathbb{C}((Z, \chi))$

$\rho_{j, Z_{\nu}^{\prime}}^{\prime}(H(Z), \bar{H}(\chi, \bar{Q}(\chi, Z)))=\sum_{\ell=1}^{N^{\prime}-\kappa} X_{\ell}^{v}(Z) \rho_{j, Z_{\ell}^{\prime}}^{\prime}(H(Z), \bar{H}(\chi, \bar{Q}(\chi, Z)))$,

which proves (ii). The proof of the proposition is now complete.

\section{Two convergence results for formal CR maps}

In this section, we prove two convergence results for formal CR maps. The first one establishes in full generality the convergence of holomorphically nondegenerate formal maps as previously defined. The second convergence result tackles the case of holomorphically degenerate maps.

Theorem 5.1 Let $M, M^{\prime}$ be real-analytic generic submanifolds respectively in $\mathbb{C}^{N}$ and $\mathbb{C}^{N^{\prime}}$ through the origin and $H:\left(\mathbb{C}^{N}, 0\right) \rightarrow\left(\mathbb{C}^{N^{\prime}}, 0\right)$ be a formal holomorphic map. Assume that $M$ is of finite type and that $H$ is a holomorphically nondegenerate map. Then $H$ is convergent.

This result by itself is completely new. It implies a number of existing non-trivial convergence results in the literature such as the convergence of formal maps of generic full rank between holomorphically nondegenerate generic submanifolds of finite type of the same dimension $[6,27]$ as well as the convergence of so-called essentially finite mappings between generic submanifolds in complex space of different dimension [23, Theorem 7.1]. 
Proof of Theorem 5.1 We use the notation previously introduced in Sect. 3.2 and in Sect. 4. As $H$ is a holomorphically nondegenerate formal map, we may find $N^{\prime}$ multi-indices $\alpha^{1}, \ldots, \alpha^{N^{\prime}} \in \mathbb{N}^{n}$ and $N^{\prime}$ integers $j_{1}, \ldots, j_{N^{\prime}} \in$ $\left\{1, \ldots, d^{\prime}\right\}$ such that the $N^{\prime}$ vectors $E_{j_{r}}^{\alpha^{r}}, 1 \leq r \leq N^{\prime}$, given in (4.2), have rank $N^{\prime}$ over $\mathbb{C}((M))$. Note that for every $1 \leq r \leq N^{\prime}$, we may write

$$
\bar{L}^{\alpha^{r}} \rho_{j_{r}}^{\prime}(H(Z), \overline{H(Z)})=: \Theta_{r}(Z, \bar{Z}, \overline{G(Z)}, H(Z)),
$$

for some formal holomorphic power series mapping $G$ and some convergent power series $\Theta_{r}$ of its four arguments. As $H$ sends $M$ into $M^{\prime}$, writing $\Theta=$ $\left(\Theta_{1}, \ldots, \Theta_{N^{\prime}}\right)$, we have

$$
\left.\Theta(Z, \bar{Z}, \overline{G(Z)}, H(Z))\right|_{M}=0 .
$$

Furthermore the rank assumption on the above vectors $E_{j_{r}}^{\alpha^{r}}, 1 \leq r \leq N^{\prime}$, means that

$$
\left.\operatorname{det} \frac{\partial \Theta}{\partial Z^{\prime}}(Z, \bar{Z}, \overline{G(Z)}, H(Z))\right|_{M} \not \equiv 0 .
$$

Applying Proposition 3.1, we get that $H$ is convergent, which completes the proof of the theorem.

The next theorem provides a sufficient condition for the convergence of a holomorphically degenerate formal map in terms of the Levi-form of the target manifold.

Theorem 5.2 Let $M, M^{\prime}$ be real-analytic generic submanifolds respectively in $\mathbb{C}^{N}$ and $\mathbb{C}^{N^{\prime}}$ through the origin and $H:\left(\mathbb{C}_{Z}^{N}, 0\right) \rightarrow\left(\mathbb{C}_{Z^{\prime}}^{N^{\prime}}, 0\right)$ be a formal holomorphic map. Assume that $M$ is of finite type, that $H$ is a holomorphically degenerate map of generic degeneracy $\kappa>0$ and let $\rho^{\prime}=\left(\rho_{1}^{\prime}, \ldots, \rho_{d^{\prime}}^{\prime}\right)$ and $d^{\prime}$ be given as in Sect. 4. Assume that for every $\kappa$-tuple $\left(V^{1}, \ldots, V^{\kappa}\right)$ of $\mathbb{C}((Z))$ linearly independent formal meromorphic infinitesimal deformations of $H$, $V^{j}=\left(V_{1}^{j}, \ldots, V_{N^{\prime}}^{j}\right) \in(\mathbb{C}((Z)))^{N^{\prime}}$, the $\kappa \times\left(\kappa d^{\prime}\right) \mathbb{C}((M))$-valued matrix $\mathscr{C}=\left[\mathscr{C}^{1}\left|\mathscr{C}^{2}\right| \ldots \mid \mathscr{C}^{d^{\prime}}\right]$ given by $\mathscr{C}^{\ell}=\left(\mathscr{C}_{\nu \gamma}^{\ell}\right), 1 \leq v, \gamma \leq \kappa$, where

$$
\mathscr{C}_{\nu \gamma}^{\ell}=\left.\sum_{1 \leq i, j \leq N^{\prime}}\left\{V_{i}^{\nu}(Z) \overline{V_{j}^{\gamma}(Z)} \frac{\partial^{2} \rho_{\ell}^{\prime}}{\partial Z_{i}^{\prime} \bar{Z}_{j}^{\prime}}(H(Z), \overline{H(Z)})\right\}\right|_{M},
$$

is of rank $\kappa$ over $\mathbb{C}((M))$. Then $H$ is convergent.

Proof of Theorem 5.2 As $H$ is a holomorphically degenerate map of generic degeneracy $\kappa>0$, we may find find $N^{\prime}-\kappa$ multi-indices $\alpha^{1}, \ldots, \alpha^{N^{\prime}-\kappa} \in$ $\mathbb{N}^{n}$ and $N^{\prime}-\kappa$ integers $j_{1}, \ldots, j_{N^{\prime}-\kappa} \in\left\{1, \ldots, d^{\prime}\right\}$ such that the $N^{\prime}-\kappa$ 
vectors $E_{j_{r}}^{\alpha^{r}}, 1 \leq r \leq N^{\prime}-\kappa$, given in (4.2), have rank $N^{\prime}-\kappa$ over $\mathbb{C}((M))$. Furthermore, interchanging the $Z^{\prime}$-coordinates if necessary, we may split $Z^{\prime}=$ $\left(\widetilde{Z}^{\prime}, \widehat{Z}^{\prime}\right) \in \mathbb{C}^{N^{\prime}-\kappa} \times \mathbb{C}^{\kappa}$ so (i) and (ii) in Proposition 4.4 hold. We therefore have the following identities:

$$
\begin{cases}\left.\bar{L}^{\alpha^{r}} \rho_{j_{r}}^{\prime}(H(Z), \overline{H(Z)})\right|_{M}=0, & r=1, \ldots, N^{\prime}-\kappa, \\ \left.\sum_{q=1}^{N^{\prime}}\left(X_{q}^{v}(Z) \rho_{j, Z_{q}^{\prime}}^{\prime}(H(Z), \overline{H(Z)})\right)\right|_{M}=0, & N^{\prime}-\kappa+1 \leq v \leq N^{\prime}, 1 \leq j \leq d^{\prime},\end{cases}
$$

where

$$
X_{i}^{v}=\delta_{i v}, \quad N^{\prime}-\kappa+1 \leq i, v \leq N^{\prime} .
$$

Taking complex conjugates of the second identity in (5.4) and using that each component of $\rho^{\prime}$ is real-valued, we obtain the following system:

$$
\begin{cases}\left.\bar{L}^{\alpha^{r}} \rho_{j_{r}}^{\prime}(H, \bar{H})\right|_{M}=0, & r=1, \ldots, N^{\prime}-\kappa, \\ \left.\rho_{j, \bar{Z}_{v}^{\prime}}^{\prime}(H, \bar{H})\right|_{M}+\left.\sum_{q=1}^{N^{\prime}-\kappa} \overline{X_{q}^{v}} \rho_{j, \bar{Z}_{q}^{\prime}}^{\prime}(H, \bar{H})\right|_{M}=0, & N^{\prime}-\kappa+1 \leq v \leq N^{\prime}, 1 \leq j \leq d^{\prime} .\end{cases}
$$

Multiplying the second set of equations of (5.6) by a suitable nonzero power series of the form $\overline{D(Z)}$, we see that $H$ satisfies a system of complex-analytic equations of the form $\left.\mathscr{S}(Z, \bar{Z}, \overline{G(Z)}, H(Z))\right|_{M}=0$ for some formal holomorphic power series mapping $G$ and some $\mathbb{C}^{N^{\prime}-\kappa+d^{\prime} \kappa}$-valued convergent power series mapping $\mathscr{S}$ of its arguments. We shall now prove that we can extract $N^{\prime}$ components $\mathscr{S}^{\#}$ of the mapping $\mathscr{S}$ so that

$$
\left.\operatorname{det} \frac{\partial \mathscr{S}^{\#}}{\partial Z^{\prime}}(Z, \bar{Z}, \overline{G(Z)}, H(Z))\right|_{M} \not \equiv 0
$$

which will allow us to apply Proposition 3.1 to conclude that $H$ is convergent.

To this aim, we shall determine the Jacobian matrix $\mathscr{J}$ (with respect to $Z^{\prime}$ ) of the system (5.6) and show that its rank (over $\mathbb{C}((M))$ ) is equal to $N^{\prime}$. Such a matrix has its coefficients in $\mathbb{C}((M))$. For convenience of notation we set $\alpha^{0}=0 \in \mathbb{N}^{n}$ and

$$
T_{\ell, j}^{r}:=\left.\bar{L}^{\alpha^{r}} \rho_{j, Z_{\ell}^{\prime}}^{\prime}(H, \bar{H})\right|_{M}, 0 \leq r \leq N^{\prime}-\kappa, 1 \leq j \leq d^{\prime}, 1 \leq \ell \leq N^{\prime} .
$$

We have 


$$
\mathscr{J}^{T}=\left[\begin{array}{ccc|c|c|c}
T_{1, j_{1}}^{1} & \ldots & T_{1, j_{N^{\prime}-\kappa}}^{N^{\prime}-\kappa} & \left(C_{\beta, \gamma}^{1}\right)_{\substack{1 \leq \beta \leq N^{\prime}-\kappa, N^{\prime}-\kappa+1 \leq \gamma \leq N^{\prime}}} & \ldots & \left(C_{\beta, \gamma}^{d^{\prime}}\right)_{\substack{1 \leq \beta \leq N^{\prime}-\kappa, N^{\prime}-\kappa+1 \leq \gamma \leq N^{\prime}}} \\
\vdots & \ldots & \vdots & & \\
T_{N^{\prime}-\kappa, j_{1}}^{1} & \cdots & T_{N^{\prime}-\kappa, j_{N^{\prime}-\kappa}}^{N^{\prime}-\kappa} & & \\
\hline T_{N^{\prime}-\kappa+1, j_{1}}^{1} & \cdots & T_{N^{\prime}-\kappa+1, j_{N^{\prime}-\kappa}}^{N^{\prime}-\kappa} \\
\vdots & \cdots & \vdots \\
T_{N^{\prime}, j_{1}}^{1} & \cdots & T_{N^{\prime}, j_{N^{\prime}-\kappa}}^{N^{\prime}-\kappa} & \left(C_{\beta, \gamma}^{1}\right)_{\substack{N^{\prime}-\kappa+1 \leq \beta \leq N^{\prime} \\
N^{\prime}-\kappa+1 \leq \gamma \leq N^{\prime}}} & \ldots & \left(C_{\beta, \gamma}^{d^{\prime}}\right)_{\substack{N^{\prime}-\kappa+1 \leq \beta \leq N^{\prime}, N^{\prime}-\kappa+1 \leq \gamma \leq N^{\prime}}}
\end{array}\right]
$$

where for $1 \leq k \leq d^{\prime}, 1 \leq \beta \leq N^{\prime}$ and $N^{\prime}-\kappa+1 \leq \gamma \leq N^{\prime}$,

$$
C_{\beta, \gamma}^{k}=\left.\sum_{q=1}^{N^{\prime}} \overline{X_{q}^{\gamma}} \rho_{k, Z_{\beta}^{\prime} \bar{Z}_{q}^{\prime}}(H, \bar{H})\right|_{M}
$$

Note that if we apply each differential operator $\bar{L}^{\alpha^{r}}, r=0, \ldots, N^{\prime}-\kappa$, to the second identity in (5.4), we get, using (5.5),

$$
T_{\nu, j}^{r}+\sum_{q=1}^{N^{\prime}-\kappa} X_{q}^{\nu} T_{q, j}^{r}=0, \quad N^{\prime}-\kappa+1 \leq v \leq N^{\prime}, 1 \leq j \leq d^{\prime}
$$

as a power series identity in $\mathbb{C}((M))$. Denote by $\mathscr{L}_{v}$ the $v$-th row of the matrix $\mathscr{J}^{T}$. Replacing $\mathscr{L}_{v}$ by $\mathscr{L}_{v}+\sum_{q=1}^{N^{\prime}-\kappa} X_{q}^{v} \mathscr{L}_{q}$ in the matrix $\mathscr{J}^{T}$ for $N^{\prime}-\kappa+1 \leq$ $v \leq N^{\prime}$, and using (5.8), we obtain the following matrix

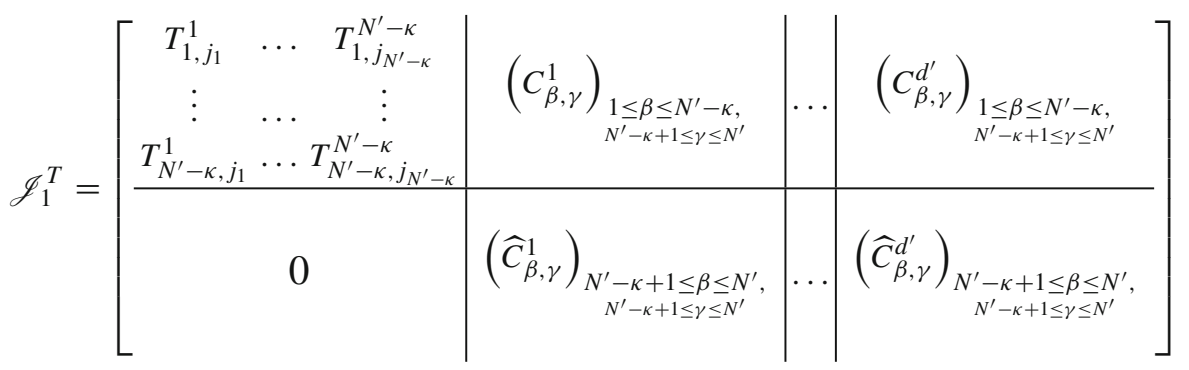

with the same rank as that of $\mathscr{J}^{T}$. Here for $1 \leq k \leq d^{\prime}, N^{\prime}-\kappa+1 \leq \beta \leq N^{\prime}$ and $N^{\prime}-\kappa+1 \leq \gamma \leq N^{\prime}$, we have, using (5.5)

$$
\widehat{C}_{\beta, \gamma}^{k}=C_{\beta, \gamma}^{k}+\sum_{q=1}^{N^{\prime}-\kappa} X_{q}^{\beta} C_{q, \gamma}^{k}=\sum_{q=1}^{N^{\prime}} X_{q}^{\beta} C_{q, \gamma}^{k}=\left.\sum_{1 \leq q, s \leq N^{\prime}} X_{q}^{\beta} \overline{X_{s}^{\gamma}} \rho_{k, Z_{q}^{\prime}}^{\prime} \bar{Z}_{s}^{\prime}(H, \bar{H})\right|_{M} .
$$


As the $\left(N^{\prime}-\kappa\right) \times\left(N^{\prime}-\kappa\right)$ matrix on the top left of $\mathscr{J}_{1}^{T}$ is invertible (because of the conclusion of Proposition 4.4 (i) that is known to hold), the rank of the matrix $\mathscr{J}^{T}$ is equal to $N^{\prime}$ if and only if the matrix

$$
\left[\begin{array}{l|l}
\left(\widehat{C}_{\beta, \gamma}^{1}\right)_{\substack{N^{\prime}-\kappa+1 \leq \beta \leq N^{\prime} \\
N^{\prime}-\kappa+1 \leq \gamma \leq N^{\prime}}} & \ldots \mid\left(\widehat{C}_{\beta, \gamma}^{d^{\prime}}\right)_{\begin{array}{r}
N^{\prime}-\kappa+1 \leq \beta \leq N^{\prime}, \\
N^{\prime}-\kappa+1 \leq \gamma \leq N^{\prime}
\end{array}}
\end{array}\right]
$$

is of rank $\kappa$. Since the $\kappa$ vectors $\left(X^{N^{\prime}-\kappa+1}, \ldots, X^{N^{\prime}}\right)$ are $\mathbb{C}((Z))$-linearly independent vectors of $\mathbb{C}((Z))^{N^{\prime}}$ and are tangent to $M^{\prime}$ along $H(M)$ in view of (5.5) and (5.4), the conclusion of Theorem 5.2 follows from (5.9). The proof is complete.

\section{Proof of Theorem 1.1}

For the proof of Theorem 1.1, we will need the following result for strongly pseudoconvex generic submanifolds. Recall that a generic submanifold $M^{\prime} \subset$ $\mathbb{C}^{N^{\prime}}$ is strongly pseudoconvex at a point $p_{0} \in M^{\prime}$ if $M^{\prime}$, near $p_{0}$, is contained in some strongly pseudoconvex real hypersurface of $\mathbb{C}^{N^{\prime}}$.

Lemma 6.1 Let $M, M^{\prime}$ be real-analytic generic submanifolds respectively in $\mathbb{C}^{N}$ and $\mathbb{C}^{N^{\prime}}$ through the origin and $H:\left(\mathbb{C}_{Z}^{N}, 0\right) \rightarrow\left(\mathbb{C}_{Z^{\prime}}^{N^{\prime}}, 0\right)$ be a formal holomorphic map. Assume that $M^{\prime}$ is strongly pseudoconvex near 0 and let $\rho^{\prime}=\left(\rho_{1}^{\prime}, \ldots, \rho_{d^{\prime}}\right)$ be a real-analytic $\mathbb{R}^{d^{\prime}}$-valued defining function for $M^{\prime}$ near 0 where $d^{\prime}=\operatorname{codim} M^{\prime}$. Then, for every $\kappa \in\left\{1, \ldots, N^{\prime}\right\}$ and for every $\kappa$-uple $\left(V^{1}, \ldots, V^{\kappa}\right)$ of $\mathbb{C}((Z))$-linearly independent formal meromorphic infinitesimal deformations of $H$, the associated matrix $\mathscr{C}=\left[\mathscr{C}^{1}\left|\mathscr{C}^{2}\right| \ldots \mid \mathscr{C}^{d^{\prime}}\right]$ given by (5.3) is of rank $\kappa$ over $\mathbb{C}((M))$.

Proof As $M^{\prime}$ is strongly pseudoconvex, there exists $\left(\mu_{1}, \ldots, \mu_{d^{\prime}}\right) \in \mathbb{R}^{d^{\prime}} \backslash\{0\}$ such that the complex hessian of $\rho^{\#}:=\sum_{i=1}^{d^{\prime}} \mu_{i} \rho_{i}^{\prime}$ at 0 is positive definite on $T_{0}^{c} M^{\prime}$, the complex tangent space of $M^{\prime}$ at 0 (see e.g. [13]). Let $\kappa \in\left\{1, \ldots, N^{\prime}\right\}$ and $\left(V^{1}, \ldots, V^{\kappa}\right)$ be $\mathbb{C}((Z))$-linearly independent formal meromorphic infinitesimal deformations of $H$. We shall prove that the $\mathbb{C}((M))$ valued matrix $\mathscr{G}=\left(\mathscr{G}_{\nu \gamma}\right)_{1 \leq \nu, \gamma \leq \kappa}$ given by

$$
\mathscr{G}_{\nu \gamma}=\left.\sum_{1 \leq i, j \leq N^{\prime}} V_{i}^{\nu}(Z) \overline{V_{j}^{\gamma}(Z)} \frac{\partial^{2} \rho^{\#}}{\partial Z_{i}^{\prime} \bar{Z}_{j}^{\prime}}(H(Z), \overline{H(Z)})\right|_{M},
$$

is invertible in $\mathbb{C}((M))$, which, as the reader can check, clearly implies the desired conclusion. Since there exists a non-zero formal power series $S(Z) \in$ 
$\mathbb{C}[[Z]]$ such that every component of the vector $S V^{v}, v=1, \ldots, \kappa$, belongs to $\mathbb{C}[[Z]]$, we may assume that each vector $V^{v} \in(\mathbb{C}[[Z]])^{N^{\prime}}$. Assume, by contradiction, that $\mathscr{G}$ is not invertible in $\mathbb{C}((M))$. Then there exists a non-zero vector $U=\left(U_{1}, \ldots, U_{\kappa}\right) \in(\mathbb{C}[[M]])^{\kappa}$ such that

$$
\mathscr{G} \cdot U^{T}=0
$$

Setting $W=\left.\sum_{\nu=1}^{\kappa} U_{v} V^{v}\right|_{M} \in(\mathbb{C}[[M]])^{N^{\prime}}$, we get from (6.1) and the fact that the vectors $V^{j}$ are tangent to $M^{\prime}$ along $H(M)$ that

$$
\left.\sum_{q=1}^{N^{\prime}} W_{q} \rho_{j, Z_{q}^{\prime}}^{\prime}(H, \bar{H})\right|_{M}=0,1 \leq j \leq d^{\prime},\left.\quad \sum_{1 \leq i, j \leq N^{\prime}} W_{i} \overline{W_{j}} \frac{\partial^{2} \rho^{\#}}{\partial Z_{i}^{\prime} \bar{Z}_{j}^{\prime}}(H, \bar{H})\right|_{M}=0 .
$$

If $W$ were not identically zero (in $\mathbb{C}[[M]]$ ), one could find a real-analytic curve $\Gamma:\left(\mathbb{R}_{t}, 0\right) \rightarrow(M, 0)$ such that $(W \circ \Gamma)(t) \not \equiv 0$ in $\mathbb{C}[[t]]^{N^{\prime}}$. Hence we could write $(W \circ \Gamma)(t)=t^{m} \omega+O\left(t^{m+1}\right)$ for some non zero vector $\omega \in \mathbb{C}^{N^{\prime}}$ and some integer $m \geq 1$. From (6.2) we must have that $\omega \in T_{0}^{c} M^{\prime}$ and that it lies in the kernel of $\partial \bar{\partial} \rho^{\#}$ at 0 , which is impossible as $\left.\partial \bar{\partial} \rho^{\#}\right|_{T_{0}^{c} M^{\prime}}$ is positive definite. Hence $W$ must be identically zero, which means that the vectors $\left(V^{1}, \ldots, V^{\kappa}\right)$ are $\mathbb{C}((M))$-linearly dependent. Since $\left(V^{1}, \ldots, V^{\kappa}\right)$ are vectors in $\mathbb{C}[[Z]]^{N^{\prime}}$, it is not difficult to see that these vectors must therefore be $\mathbb{C}((Z))$-linearly dependent, reaching a contradiction. Hence $\mathscr{G}$ is invertible in $\mathbb{C}((M))$. The proof of the lemma is complete.

Proof of Theorem 1.1 Without loss of generality we may assume that $p=$ $p^{\prime}=0$. If $H$ is a holomorphically nondegenerate map, then $H$ is convergent by Theorem 5.1. If $H$ is a holomorphically degenerate map of generic degeneracy $\kappa>0$, then the convergence of $H$ follows by applying Theorem 5.2 together with Lemma 6.1. The proof is complete.

Acknowledgements Open access funding provided by University of Vienna. The authors would like to thank the referee for his remarks that helped improve the exposition of the paper.

Open Access This article is distributed under the terms of the Creative Commons Attribution 4.0 International License (http://creativecommons.org/licenses/by/4.0/), which permits unrestricted use, distribution, and reproduction in any medium, provided you give appropriate credit to the original author(s) and the source, provide a link to the Creative Commons license, and indicate if changes were made.

\section{References}

1. Artin, M.: On the solutions of analytic equations. Invent. Math. 5, 277-291 (1968) 
2. Baouendi, M.S., Ebenfelt, P., Rothschild, L.P.: Real Submanifolds in Complex Space and Their Mappings. Princeton Math. Series, vol. 47. Princeton University Press, Princeton (1999)

3. Baouendi, M.S., Ebenfelt, P., Rothschild, L.P.: Convergence and finite determination of formal CR mappings. J. Am. Math. Soc. 13(4), 697-723 (2000)

4. Baouendi, M.S., Ebenfelt, P., Huang, X.: Holomorphic maps between hyperquadrics with small signature difference. Am. J. Math. 133, 1633-1661 (2011)

5. Baouendi, M.S., Huang, X.: Super-rigidity for holomorphic mappings between hyperquadrics with positive signature. J. Diff. Geom. 69, 379-398 (2005)

6. Baouendi, M.S., Mir, N., Rothschild, L.P.: Reflection ideals and mappings between generic submanifolds in complex space. J. Geom. Anal. 12(4), 543-580 (2002)

7. Berhanu, S., Cordaro, P., Hounie, J.: An Introduction to Involutive Structures. New Mathematical Monographs, vol. 6. Cambridge University Press, Cambridge (2008)

8. Berhanu, S., Xiao, M.: On the $C^{\infty}$ version of the reflection principle for mappings between CR manifolds. Am. J. Math. 137(5), 1365-1400 (2015)

9. Chern, S.S., Moser, J.-K.: Real hypersurfaces in complex manifolds. Acta Math. 133, 219271 (1974)

10. Della Sala, G., Juhlin, R., Lamel, B.: Formal theory of Segre varieties. Ill. J. Math. 56(1), 101-125 (2012)

11. Della Sala, G., Lamel, B., Reiter, M.: Local and infinitesimal rigidity of hypersurface embeddings. Trans. Am. Math. doi:10.1090/tran/6885

12. Forstnerič, F.: Extending proper holomorphic mappings of positive codimension. Invent. Math. 95, 31-62 (1989)

13. Forstnerič, F.: Mappings of strongly pseudoconvex Cauchy-Riemann manifolds. In: Proceedings of Symposia in Pure Mathematics, vol. 52, pp. 59-92 (1991)

14. Huang, X.: Geometric analysis in several complex variables. Ph.D. Thesis, Washington University, St Louis (1994)

15. Huang, X.: On the mapping problem for algebraic real hypersurfaces in the complex spaces of different dimensions. Ann. Inst. Fourier 44, 433-463 (1994)

16. Huang, X., Zhang, Y.: On the CR transversality of holomorphic maps into hyperquadrics. In: Complex geometry and dynamics. Abel Symposia, vol. 10, pp. 139-155. Springer, Cham (2015)

17. Juhlin, R.: Determination of formal CR mappings by a finite jet. Adv. Math. 222, 1611-1648 (2009)

18. Lamel, B.: Holomorphic maps of real submanifolds in complex spaces of different dimensions. Pac. J. Math. 201(2), 357-387 (2001)

19. Meylan, F., Mir, N., Zaitsev, D.: Approximation and convergence of formal CR mappings. Int. Math. Res. Not. 4, 211-242 (2003)

20. Meylan, F., Mir, N., Zaitsev, D.: On some rigidity properties of mappings between CR-submanifolds in complex space, Journees Equations aux derivees partielles, Ecole Polytechnique (Forges-Les-Eaux, Juin 2003), Exposé XII (2003)

21. Mir, N.: Formal biholomorphic maps of real-analytic hypersurfaces. Math. Res. Lett. 7, 343-359 (2000)

22. Mir, N.: Convergence of formal embeddings between real-analytic hypersurfaces in codimension one. J. Diff. Geom. 62, 163-173 (2002)

23. Mir, N.: On the convergence of formal mappings. Comm. Anal. Geom. 10(1), 23-59 (2002)

24. Mir, N.: Holomorphic deformations of real-analytic CR maps and analytic regularity of CR mappings. J. Geom. Anal. 27(3), 1920-1939 (2017). doi:10.1007/s12220-016-9745-4

25. Rothschild, L.P.: Mappings Between Real Submanifolds in Complex Space, Explorations in Complex and Riemannian Geometry, Contemporary Mathematics, vol. 332. American Mathematical Society, Providence (2003) 
26. Stanton, N.: Infinitesimal CR automorphisms of real hypersurfaces. Am. J. Math. 118, 209-233 (1996)

27. Sunyé, J.-C.: On formal maps between generic submanifolds in complex space. J. Geom. Anal. 19, 944-962 (2009) 